\title{
Wenn sich Geschichte und Fantastik begegnen: Nachts unter der steinernen Brücke von Leo Perutz
}

When history meets fantastic: By night under the stone bridge from Leo Perutz

Ricarda Hirte

Universidad de Córdoba

ricarda.hirte@uco.es

Orcid: https://orcid.org/0000-0002-8582-1259

Recibido: 02/09/20212

Aceptado: 22/12/2021

DOl: https://dx.doi.org/10.12795/mAGAzin.2021.i29.04

\section{Zusammenfassung:}

Leo Perutz' Roman Nachts unter der steinernen Brücke ist in einem Zwischenbereich von Geschichte und Fantastik angesiedelt. Es ist somit nicht leicht, diesen Roman klar in eine Literaturgattung einzugliedern und diese Eigenschaft bereitete Perutz bei seiner Erstveröffentlichung auch einige Probleme: So konnte er diesen Roman nicht in seinem bevorzugten Verlag in Wien veröffentlichen, sondern er erschien in einem, der bald darauf finanzielle Probleme hatte, weswegen der Roman nur langsam bekannt wurde. Da Perutz den Roman nach dem Zweiten Weltkrieg fertigstellte, sah sein Verlag das Thema als zu jüdisch an, da man davon ausging, dass die Gesellschaft nach den Schrecken des Krieges nicht an diesen Themen interessiert war. Perutz verteidigte sich und sah seinen Roman als einen rein historischen an. Beide Ansichten haben Rechtsgültigkeit, und sowohl das Jüdische wie die Geschichte sind Hauptbestandteile des Romans. Die Frage stellt sich allerdings, wie diese Themen verarbeitet worden sind und inwiefern sie sich gegenseitig bedingen.

Schlüsselwörter: Geschichte, Fantastik, Transkulturalität, Realität, Irrealität

\begin{abstract}
:
Leo Perutz's novel By night under the stone bridge is situated in an intermediate area between history and fantastic. It is thus not easy to clearly classify this novel into a literary genre, and this characteristic also caused Perutz some problems when it was first published: for example, he could not publish this novel in his preferred publishing house in Vienna, but it appeared in one that had financial problems soon after, which is why the novel became known only insidiously. As Perutz completed the novel after the Second World War, his publisher saw the subject as too Jewish, as they found that society was not interested in these topics after the horrors of the war. Perutz defended himself and saw his novel as a purely historical one. Both views have legal validity and both Jewish elements and history are main components of the novel. However, the question arises as to how these themes were processed and to what extent they are mutually dependent.
\end{abstract}

Keywords: history, fantastic, transculturality, reality, unreality 


\section{Einleitung}

Die literarische Einordnung von Leo Perutz Roman Nachts unter der steinernen Brücke ist nicht ganz einfach, da der Autor die 14 Erzählungen zu einem Roman zusammenfasste, der unter historischen wie auch fantastischen Prämissen gelesen werden kann, zumeist aber als mythische Legenden des Alten Prags aufgefasst werden. Geschickt verknüpft Perutz historische Ereignisse, die normalerweise in keinem Geschichtsbuch zu finden sind, mit fantastischen Elementen, so dass der Wahrheitsgehalt des Romans zwischen wirklichmöglich und unrealistisch-unmöglich schwankt. Der Roman kann hinsichtlich seiner Komposition in zwei verschiedenen Richtungen interpretiert werden: die des historischen Romans und die des fantastischen. Beide Richtungen beanspruchen für sich, einer Literaturgattung, zumindest aber einem unabhängigen Genre, anzugehören. Dies impliziert, dass beide Richtungen nicht komplementär, sondern isoliert betrachtet werden müssen. Dass dem nicht so ist, will der Beitrag beweisen.

\subsection{Geschichte und Fantastik als literarische Gattung}

Auf den ersten Blick stehen sich Geschichtlichkeit und Phantastik gegenüber und können nicht verbunden werden. Die Geschichte ist immer Teil der Vergangenheit und sie bezieht sich im Allgemeinen auf Ereignisse, die nicht ins Vergessen geraten sollen; sie wird gedeutet und anhand ihrer, versucht man den zeitlichen Wandel zu ergründen, um Aussagen über die Gegenwart und die Zukunft machen zu können. Geschichte beginnt im Jetzt und projektiert sich immer rückläufig. Geschichte ist aber auch unsere menschheitliche Historie und ist ein Konstituens der Kultur, denn wie Luhmann feststellt ist das Anliegen der Kultur «das Beobachten von Beobachtern und um eine bestimmte Form für die Frage, wie Beobachter Beobachter beobachten» (Luhmann 1995: 32). Und beobachten hat wiederum mit Geschichtlichkeit zu tun, denn wie Luhmann weiter erklärt ist Kultur «das Gedächtnis sozialer Systeme, vor allem des Gesellschaftssystems. Kultur ist, anders gesagt, die Sinnform der Rekursivität sozialer Kommunikation» (Luhmann 1995: 47). Luhmann konzentriert sich auf den Vergleich, wenn er Kultur definieren möchte. Hierbei sind folgende Überlegungen anzuführen: Wenn man davon ausgeht, dass Geschichte und Kultur auf gemeinsamen Eckpfeilern ruhen, muss ein Vergleich immer von zwei Punkten ausgehen, da ansonsten ein Vergleichen unmöglich wäre. Man könnte anführen, dass es immer verschiedene Sichtweisen gibt, die die Vergleichsvariante ermöglichen, allerdings vergisst man dann, dass Geschichte meistens von den Siegern und nicht von den Besiegten festgehalten wird. Diese Problematik versucht Baecker mit seiner Interpretation des Kulturbegriffs zu lösen: «Die Kultur [...] verhält sich zur Gesellschaft wie der Text zur Kommunikation» (Baecker 2013: 141). Seine Aussage löst nicht das Dilemma des geschichtlichen Wahrheitsgehalts der vermeintlichen Quellen, auf die sich die Geschichte beruft, schlägt aber eine Brücke zwischen Kultur und Geschichte, als ein sich gegenseitig bedingendes Konstituens.

Der historische Roman als Genre ist von seinem Charakter her ein fiktionales Prosawerk. Was historisch ist, ist der Handlungsrahmen, der in eine historische Zeit platziert ist, ohne Anspruch auf wissenschaftlich eruierten Wahrheitsgehalt zu erheben, wobei die Referenten Zeit, Raum und Personen sind. Schönhaar definiert ihn als einen «Romantypus, der historische Gestalten und Vorfälle behandelt oder doch in historisch beglaubigter Umgebung spielt und auf einem bestimmten Geschichtsbild beruht» (Schönhaar 1990: 201). Der historische Roman etabliert sich ab dem 19. Jahrhundert in Europa und geht einher mit dem beschriebenen Epochenbruch, der bei Foucault und anderen wie Koselleck und Luhmann artikuliert wurde. Foucault folgend, wird das bis zum 19. Jahrhundert herrschende klassische Repräsentationsmodell (Descartes) zugunsten der Geschichtlichkeit aufgeben, das heißt, wie Nöth es ausdrückt, «die Ordnung der Dinge nun nicht mehr in der Ratio und ihren Repräsentationen begründet [ist], sondern in historischen Gesetzmäßigkeiten, die dem System der Dinge selbst inhärent sind» (Nöth 2000: 167). Und weiter heißt es bei Foucault: 
Die ersten Philologen hatten sich [...] in der historischen Tiefe der Sprache die Möglichkeit des Diskurses und der Grammatik gesucht. Dadurch selbst hat die Repräsentation aufgehört, für die [...] Wörter Geltung als ihre Ursprungsart und ursprünglicher Sitz der Wahrheit zu haben [...] Die Repräsentation, die man sich von den Dingen macht, $[\ldots]$ ist der Anschein einer Ordnung, die jetzt den Dingen selbst und ihrem inneren Gesetz zugehört ${ }^{1}$ (Nöth 2002: 167).

Um den Aussagecharakter Foucaults besser folgen zu können, wird auf die Erklärung von Frank zurückgegriffen:

Die klassische Episteme gründet ja auf der Voraussetzung, dass eine vollkommene Auflösung des signifiant im signifié gelingt: Nichts am Zeichen widersteht dem Gedanken, der sich vermittels seiner repräsentiert; zumal dann nicht, wenn die Ordnung der Gedanken in ihrer Wahrheit als unzeitlich gedacht wird: Wahr ist nämlich nach klassischem Denken etwas [...], weil es schlechterdings nicht anders [...] angesehen werden kann [...] Diese Prämisse tritt außer Kraft, wenn in die Synthesis der Repräsentation die Zeit [...] interveniert (Nöth 2002: 167).

Für den historischen Roman bedeutet dies, dass er mit dem Epochenbruch ins Interesse der Autoren rückt, da er geschichtliche Ereignisse neu oder anders darstellen kann. Die Geschichte verbindet sich somit auf Romanebene mit humanistischen Zügen, gestaltet geschichtliche Fakten neu, ändert ab und verknüpft anthropologisches Wissen mit «idealistischen und materialistischen Varianten» (Müller 1994: 14) der Geschichtsphilosophie. Dieses Verfahren ist nicht immer unverfänglich, da der Abstraktionsgrad geschichtlicher Daten und/oder die Fiktionalität des Autors darüber entscheiden, wie hoch der Wahrheitsgehalt des Romans ist, damit die geschichtlichen Daten oder Ereignisse mit der Romanhandlung zusammenfallen. Da der Roman wie die Geschichte als solche immer ein Konstrukt ist, unterliegt jeder historischer Roman einer genaueren Prüfung hinsichtlich des Wahrheitsgehalts. Dennoch

[handelt es sich] beim historischen Roman um ein ästhetisch vermitteltes sozio-kulturelles Deutungsmuster, dessen Textintensionalität weder in einem historischen So-ist-es-eigentlich-gewesen, noch in einer puren Einkleidung aktueller Erfahrungsmomente des Autors aufgehen kann (Müller 1994: 15).

Man könnte fragen, was ist eigentlich Geschichte, wie lässt sich diese dingbar machen, aber dies würde den Untersuchungsrahmen sprengen. Zusammenfassend kann man nach Müller sagen, dass

der historische Roman [...] also ein perspektivisch gebundenes, poetisch Angeordnetes Konstrukt mit Akzentuierung situativer-belegbarer-Konstellationen im Spannungsfeld zwischen Fiktion und Referentialisierbarkeit [ist], das von einem bestimmten Autor zu einem bestimmten Zeitpunkt für ein bestimmtes/unbestimmtes Publikum geschrieben worden ist (Müller 1994: 17).

Geschichte und Historizität ist auch bei Perutz ein zentrales Thema, bevor allerdings auf den Roman eingegangen wird, muss der Aspekt des Fantastischen als Gegenpol zum Historischen angesprochen werden. Das Fantastische hat innerhalb der Literatur eine lange Tradition und seine Ausdrucksformen lassen sich bis in die Antike in literarischen Texten ausmachen. Für eine ausführliche Phantasma-Genesis ist hier kein Raum, doch war es E.T.A. Hoffmann, der der Fantastik einen Platz in der Literatur eingeräumt hat, die sich zu einer Literaturgattung entwickelt hat. Dementsprechend gibt es auch eine Theoriegeschichte, die versucht, die Fantastik in verschiedenen Textsorten zu definieren. Mit der von Todorov (2013) entwickelten Definition des Fantastischen ist hinsichtlich einer Phantasma-Genesis ein Punkt erreicht worden, zu dem ältere Definitionen hinführen und von dem neuere Definitionen ausgehen. Demnach entstellt das Fantastische die Kategorien von Zeit, Raum und Kausalität und ist daher weder mit den Normen der mimetischen Konvention erklärbar noch respektiert sie die ästhetischen Prinzipien der Angemessenheit und der Ähnlichkeit. Der Begriff der Fantastik ist durch Todorov zwar erklärt, doch fehlt ihm zur besseren Definierung ein weiterer Aspekt: Fantastische Elemente, kurz Phantasmas, und somit die Fantastik rekurriert immer auf einen kulturellen Kontext, das heißt, dass die Fantastik anthropologische Aussagen formuliert. Anthropologisches Wissen wiederum ist immer an 
kulturologisches Wissen gekoppelt. Die kulturologische Komponente beansprucht für sich zwei Aspekte: Jede Kultur besitzt einen Bereich des Verdrängten, Vergessenen, Tabuisierten, zusammengefasst in dem Fremden, das es nach der herrschenden kulturellen Norm auszugrenzen gilt. Die kulturelle Ordnung manifestiert sich somit in der Opposition von Fremd-Eigen. Ein fantastischer Text thematisiert den Bereich des Fremden, des Nicht-Eigenen einer Kultur, holt somit verloren gegangenes Kulturgut zurück, die den Ausgrenzungen zum Opfer gefallen sind, und provoziert gleichermaßen Unordnung. Unordnung verstanden in dem Sinne, dass das Reale und damit die Präsenz einer funktionierenden Kultur in Frage gestellt wird. Die Fantastik, und in der ihr innewohnenden Irrealität, reibt sich an den Kategorien des Realen. Ein weiterer Aspekt ist die Beziehung zwischen dem Text, seiner Entstehungszeit und den vorkommenden fantastischen Elementen. Diese drei Punkte sind für die Definierung eines fantastischen Textes wichtig, da sich die Fantastik nur anhand der Kultur und ihren Definitionen als solche herleiten lässt. Dies bedeutet, dass ein Text immer in seinem Entstehungskontext gelesen und klassifiziert werden muss, denn das Wissen und das Eigene einer Kultur verändert sich, und ein Text kann in verschiedenen Epochen gelesen, als ein realistischer oder aber auch als ein fantastischer eingestuft werden, je nach seinem historischen Kontext. Zu besseren Veranschaulichung sei ein Beispiel angeführt: In der Kultur des Mittelalters wurde von der realen Existenz von Hexen und Magiern ausgegangen, das heißt, dass die Menschen in dieser Zeit an ihre Existenz, an die Magie und ihre Wirkung glaubten. Liest man einen Text aus heutiger Sicht, so kommen dem Leser hinsichtlich der Wirklichkeit, der Realität Zweifel, da er mit einem anderen kulturellen Wissen, das auch wissenschaftliches Wissen einschließt, an den Text herangeht. Trotzdem, der Text wurde in seinem Kontext geschrieben und kann Realitätsanspruch erheben, da er in seinem kulturellen Rahmen entstand, in dem von der Wirklichkeit dieser Erscheinungen ausgegangen worden ist. Allerdings, um das Fantastische herauszustellen, bedarf es immer eines Ausgangspunkts, des Realen, denn das Unmögliche, Irreale und Gegenrationale (Charaktere des Fantastischen) ist kontrapunktisch zum Möglichen, Realen und Rationalen. Lachmann hat dies durch «Die Phantastik erzählt die Begegnung der Kultur mit ihrem Vergessen» (Lachmann 2002: 11) ausgedrückt (vgl. Hirte: 2014a).

Den Aspekt der kulturologischen Komponente hat Marianne Wünsch (1998) in ihrer Theorie herausgearbeitet. Sie geht nicht von der Rezeptionsgeschichte der Fantastik aus, sondern erklärt und definiert die fantastische Literatur auf textimmanenter Ebene. Demnach präsentiert sich das Fantastische innerhalb einer narrativen Struktur und innerhalb dieser gibt es eine Figur, die das Phänomen, das fantastische Element, wahrnimmt, und eine Textinstanz, die das Phänomen zu erklären versucht. Das Phänomen ist zudem nicht-realitätskompatibel, wie es Wünsch nennt, das heißt, es wird von der herrschenden Kultur als ein etwas nicht wirklich Existierendes aufgefasst, wobei allerdings seine Präsenz es in die Möglichkeit einer Realität rücken kann. Da das Phänomen nicht als völlig irreal betrachtet wird, kann seine Präsenz in verschiedenen Bereichen eine Erklärung finden, und oftmals wird in diesem Zusammenhang auf den Okkultismus verwiesen. Für diese Annahme einer möglichen Erklärung muss im Text eine Instanz vorhanden sein, die implizit oder explizit dies bestätigt. Wünsch nennt diese Annahme Klassifikator der Realitätsinkompatibilität. Wünsch definiert einen fantastischen Text dahingehend, dass neben der fantastischen Struktur oder Anlage des Textes auch in der Erklärbarkeit des Phänomens sich das Fantastische bestätigt, oder im abgeschwächten Fall, nicht ausgeschlossen werden kann oder ausgeschlossen ist.

Das Fantastische ist bei Perutz verbunden mit der Kabbala, erhält durch sie seine Erklärbarkeit und ist zudem Bestandteil der Kultur. Er erzählt die Begegnung zwischen Vergangenheit, dem Tabuisierten einer Kultur und dem Jetzt; gleichzeitig ist die Kabbala zentrales Element der Transkulturalität, die der Roman von Perutz aufweist. Mit Hilfe der Kabbala verbindet er die losen historisch verifizierbaren Ereignisse, setzt sie in einen narrativen Rahmen, in dem geschichtliche Ereignisse mit allgemein kulturellem, historischem wie religiösem Glauben erklärt werden und bringt verlorengegangenes Kulturgut zurück.

\subsection{Elemente, die dem Roman eine geschichtliche oder fantastische Konnotation geben}

Die Klassifizierung des Romans in historisch oder fantastisch hatte im Moment seiner Veröffentlichung auch für Perutz Konsequenzen. Die Entstehungsgeschichte des Romans umfasst drei Perioden, die eng mit Perutz Biografie verbunden sind. Die ersten Arbeiten entstehen um 1924 und finden sich im Anfangskapitel Die Pest in der Judenstadt im Roman wieder, die Perutz schrieb, als er noch in Wien wohnte. Eine zweite Schaffensperiode 
sind die Jahre vor der Emigration nach Palästina zwischen 1936 und 1937, wo er vor allem die Romanstruktur festlegt, fertiggestellt wurde der Roman allerdings erst 1951 in Tel Aviv und 1953 wurde er noch zu Perutz Lebzeiten veröffentlicht. Da es nur sehr wenig autobiographisches Material gibt, kann nur vermutet werden, dass der Roman die Antwort auf seine tiefe Existenzkrise ist, die er nach dem Tod seiner ersten Ehefrau moralisch und finanziell durch die allgemeine Weltkrise um 1929 erlebte. In dieser Zeit fühlte sich Perutz, auch wenn er eine aseptische Haltung beibehielt, von okkultistischen Gruppen und Zirkeln angezogen, um die Leere, die der Tod seiner Ehefrau in ihm verursachte, zu füllen; um genau zu sein, er versuchte anhand des Okkultismus eine Beziehung mit ihr herstellen zu können. Nicht zu vergessen ist, dass er jüdischer, sephardischer Herkunft, ist, auch wenn er seine Religion nie aktiv ausgeübt hat und auch diese kein Thema in seiner Familie war. Durch den sich verschärfenden Antisemitismus in Europa, fühlte er sich gezwungen mit seiner Familie und mit seiner zweiten Ehefrau über Italien nach Palästina zu emigrieren. In Palästina konnte er sich nicht einleben und vermisste seinen europäischen Lebensstil. Ein Indiz dafür ist, dass er die Notizen mit in sein Exil nahm und der Roman als eine Brücke zu seiner Heimat verstanden werden kann. Die Verbindung mit Prag, seiner Heimatstadt konnte er dadurch aufrechterhalten, denn «das Bewahren und Mitnehmen eines wie auch immer fragmentarischen Vorrats von Erinnerungen an eine für ihn lebensbestimmende europäische Kultur» (Müller 2007: 344), half ihm das Trauma der Emigration und Assimilation zu kompensieren. Immer wieder gibt es kurze Anmerkungen, die den Roman mit seiner Lebenssituation verbindet: So schreibt er an Annie und Hugo Lifczis, die nach Argentinien ausgewandert waren und dank ihrer Initiative einige Werke von Perutz auf Spanisch übersetzt wurden, «dass der Schriftsteller Leo Perutz noch am Leben ist» $(i b$.$) . Es scheint, dass die dritte Schaffensperiode auch mit$ Periode der kulturellen Selbstbehauptung betitelt werden kann, die die Jahre nach seiner Ankunft in Palästina bis 1951 umfasst und die Rückkehr nach Österreich miteinschließt. Der Romanabschluss fällt mit dem Jahr (1951) zusammen, in dem Perutz und seiner Familie die österreichische Nationalität wiederanerkannt wird. Seine nun folgenden Jahre bis zum Tod 1957 waren durch die Oszillation zwischen Österreich und dem Nahen Osten geprägt. Die Kombination, die er in Nachts unter der steinernen Brücke zwischen Okkultismus, ausgedrückt in der Kabbala und Geschichtlichkeit macht, situieren den Roman zwischen historisch und fantastisch und bestimmen die Schwierigkeiten seiner Veröffentlichung nach dem Zweiten Weltkrieg vor. So schreibt er an seinen Agenten Hugo Lifczis:

Ich habe das Manuskript dem Zsolnay geschickt, aber er will die Veröffentlichung hinausschieben - obwohl es ein völlig politik- und ressentimentloser, rein historischer Roman ist, der eben zum Teil im Ghetto spielt und den hohen Rabbi Loew zu einer der Hauptfiguren hat. Es ist nichts als eine Verbeugung vor dem alten Prag [...] Aber Zsolnay schont die Empfindlichkeit jenes Wiener Gesindels, das nicht gerne daran erinnert werden will, dass es Juden gibt, gegen die es sich schlecht benommen. Ich will aber nicht warten, bis - wie Zsolnay schreibt - die deutsche Seele sich Werken jüdischen Geistesguts wiedereröffnet (Serke 1987: 278).

Der Roman erschien nicht in seinem „Traditionsverlag“ Paul Zsolnay in Wien, sondern 1953 in der Frankfurter Verlagsanstalt. Da der Verlag kurze Zeit nach der Veröffentlichung in Zahlungsschwierigkeiten geriet, sah Perutz auch seinen Roman nicht in den Buchläden. Vielleicht trug dieser Umstand auch dazu bei, dass dieser Roman erst später an Popularität gewann und nicht das Thema das ausschlaggebende Problem war.

Perutz verweist eigens auf die Geschichtlichkeit seines Romans, und indem er ihn in die Zeit Kaisers Rudolf II. verortet und ihn mit jüdischer Tradition verbindet, erhalten die fantastischen Elemente durch die Kabbala ihre Erklärbarkeit. Die Kabbala ist für einen Leser heutiger europäischer Kultur a priori ein fantastisches Element, dadurch erhält der Roman einen transkulturellen Charakter. Geschickt verbindet Perutz historische Ereignisse, setzt sie in einen narrativen Rahmen und versucht, die Geschichte durch kulturellen, geschichtlichen und religiösen Glauben zu erklären; verloren geglaubtes Kulturgut setzt er so in einen neuen Sinnzusammenhang. Denn er erzählt die Geschichte nicht wie sie im Geschichtsbuch oder in historischen Quellen steht, sondern er erzählt geschichtliche Begebenheiten, die zwar auf historische Daten verweisen, aber nicht einwandfrei beweisbar sind. Es ist ein Mund-zu-Mund erzählen, eine Erzählweise, die an die Weitergabe kulturellen Guts durch reine Mündlichkeit erinnert. Der Leser wird indirekt aufgefordert selbst die geschichtlichen Begebenheiten nachzuforschen und die im Roman beschriebene Realität zu überprüfen. Diese Annahme bestätigt sich auch im 
Aufbau des Romans, wobei der Handlungsrahmen in geschichtlich und kabbalistisch-fantastisch zweigeteilt ist.

Der historische Rahmen bezieht sich auf die Stadt Prag um 1598, allerdings ist der historische Rahmen des Erzählers im Prag um 1898 angesiedelt, zu dem Zeitpunkt, an dem mit der Sanierung des jüdischen Viertels, der Josefstadt begonnen wurde. Man kann daraus schließen, dass der historische Bezug mit der Entstehung und der Modernisierung des jüdischen Prager Ghettos korreliert und gleichzeitig auf den Autor referiert, der die Sanierung miterlebte. Allerdings ist auch zu berücksichtigen, dass diese Daten mit der Entstehung der Legenden um das alte Prag, wie die des Golems oder des Juden Meisl, eng verbunden sind und deren topografische Lage nach der Sanierung verloren ging. So ist anzumerken, dass der Grabstein des Juden Meisl auf dem jüdischen Friedhof das einzige Zeugnis ist, dass diese legendäre Figur wirklich gelebt hat. Der Erzähler vollzieht eine doppelte Rückerinnerung, einerseits auf seine Jugendjahre und andererseits auf die Zeit, in der Rudolf II. Kaiser des Heiligen Römischen Reiches war, zwischen 1576 und 1612.

Kaiser Rudolf II., der aus der Habsburger Dynastie hervorging, erklärte Prag zu seiner Residenz. Die Stadt erfuhr eine Wiederbelebung auf sozialem und kulturellem Niveau, da durch den Kaisersitz viele Deutsche nach Prag umsiedelten und der Kaiser die Wiederansiedlung der Juden in der Altstadt erlaubte. Nach einer fast ausschließ von den Hussiten geprägten Zeit erhielt Prag einen multikulturellen Charakter. Kaiser Rudolf II. hatte als König von Böhmen einen Majestätsbrief verfasst, in dem er Adeligen und Städten mit lutherischer (protestantischer) Religion die Freiheit der Religionsausübung zugestand, einschließlich des Baus von Kirchen auf königlichem Grund. Als der Erzbischof von Prag eine lutherische Kirche auf seinem Territorium schloss, stellte sich die Frage, ob sie königlichen Eigentums war oder nicht. Die Lutheraner sahen in der Diskussion die Anfänge einer Gegenreformation in Böhmen und Vertreter der protestantischen Stände warfen nach einem Schauprozess zwei kaiserliche Räte und den Sekretär aus dem Fenster, was als Zweiter Prager Fenstersturz bekannt wurde und den dreißigjährigen Krieg auslöste. Kaiser Rudolf II. wurde gestürzt und Ferdinand II. (1619-1637) bemächtigte sich dem Thron. Die böhmischen Stände erklärten allerdings Ferdinand für gestürzt und wählten den reformierten Kurfürsten Friedrich V. von der Pfalz zu ihrem König, um den Protestantismus zu garantieren. Die katholischen Mächte schlossen sich gegen diese Proklamation zusammen: der Kaiser, Spanien, der Papst, Bayern und die Liga. Die Stände ihrerseits konnten sich nicht einigen und lösten sich bald auf. Der neue König von Böhmen, der gewählte Fürst der Pfalz, konnte sein Amt nur einen Winter lang ausüben und ging als der Winterkönig in die Geschichte ein; 1620 wurde er von Tilly, dem Befehlshaber der Liga, in der Schlacht am weißen Berg bei Prag besiegt. Ferdinand V. floh aus Böhmen, und er und seine Gefolgsleute wurden $\mathrm{zu}$ tschechischen und deutschen „Rebellen“ erklärt. Als geringere Strafe mussten sie das Land verlassen, als schwereres Vergehen wurden sie hingerichtet; ihr Besitz fiel an den Kaiser. Dieser geschichtliche Abschnitt findet sich im Roman im Kapitel „Die Getreuen des Kaisers“. Mit der Offensive gegen die Protestanten oder Lutheraner wurde der Majestätsbrief gebrochen und Böhmen wurde wieder katholisch. Nach dem dreißigjährigen Krieg, der für viele europäische Städte den Verlust der Religionsfreiheit mitbrachte, wurde das barocke Prag errichtet und der tschechische Nationalismus und die tschechische Sprache verloren an Einfluss. Dieser Prozess setzte sich bis ins 18. Jahrhundert fort; 1784 wurden die vier autonomen Städte Prags - Hradschin, Kleinseite, Altstadt und Neue Stadt - vereinigt, und erst 1884 kam es in Prag zu einer Wiederbelebung der tschechischen Nation, die sich in dem Revolutionsjahr in der Teilung der Karls-Universität in eine tschechische und eine deutsche Universität manifestierte.

Nach Absteckung des geschichtlichen Hintergrunds ist der Roman in den letzten Regierungsjahren Rudolfs II. und den Anfängen des dreißigjährigen Krieges angesiedelt. Das erste Kapitel des Romans beginnt mit dem großen Kindersterben, eine Anspielung auf die Pest oder einer ähnlichen Krankheit, an der die Kinder im jüdischen Ghetto von Prag sterben. Zwar gab es in Prag verschiedene Pestwellen im 16. Jahrhundert, es kann aber nicht nachgewiesen werden, dass 1598 eine Pestepidemie die Judenstadt heimsuchte, wohl aber kann bestätigt werde, dass in diesem Jahr die Pest in der Schweiz, in Chur aktiv war (Stadtarchiv der Stadt Chur: 2021). Das Kindersterben wird im Roman mit einem Zauber kabbalistischen Ursprungs verbunden: zum Schutz der jüdischen Gemeinde vor den befürchteten Pogromen. Die Juden, auch wenn sie sich in der Josefstadt, dem so benannten jüdischen Ghetto, ansiedeln durften, lebten unter der Gunst des Kaisers und waren willkürlichen Pogromen ausgesetzt. So sah sich Rabbi Loew gezwungen einen Zauberspruch auszusprechen, um dem Wunsch 
des Kaisers nachzugehen, der sich bei einem Besuch im Ghetto in eine Jüdin verliebt hatte. Es handelte sich dabei um die Ehefrau des Juden Meisl, der allerdings der Geldgeber des Kaisers war und durch seine Stellung als kaiserlicher Geldverleiher der jüdischen Gemeinde eine Zeit des Friedens bescherte. Dieses scheinbar unlösbare Dilemma versuchte Rabbi Loew mit Hilfe der Kabbala zu lösen. Somit bedient er sich der verloren gegangenen praktischen Kabbala und sprach einen Bann über Meisls Ehefrau und dem Kaiser aus: Jede Nacht besuchen sich beide Seelen im Schlafgemach des Kaisers, symbolisiert im Rosmarin, die Frau, und der Rose, dem Kaiser, die der Rabbi am Ufer der Moldau unter der steinernen Brücke pflanzt. Das Kindersterben und der Bann stehen in direkter Verbindung, da nur eine ehebrecherische Frau dieses Sterben provozieren kann. Als der Rabbi erkennt, dass er selbst das Unglück über seine Gemeinde gebracht hat, reißt er beide Pflanzen unter der Brücke aus und

in dieser Nacht erlosch die Pest in den Gassen der Judenstadt. In dieser Nacht starb in ihrem Haus auf dem Dreibrunnenplatz die schöne Esther, die Frau des Juden Meisl. In dieser Nacht fuhr auf seiner Burg zu Prag der Kaiser des Römischen Reiches, Rudolf II., mit einem Schrei aus seinem Traum (Perutz 1991: 18).

Verschiedene Elemente werden hier angesprochen wie die Kabbala, die Schönheit einer Jüdin, der Name Esther, alles Bezüge zur jüdischen Tradition und Mythologie, die in sich fantastische Züge und Elemente besitzen. Der Roman beginnt mit dem äußeren fantastisch konnotierten Erzählrahmen und schließt auch damit, sieht man vom Epilog ab, der Bezug auf den auktorialen Erzähler nimmt: Das letzte Kapitel ist dem Diskurs der Rechtfertigung zwischen dem Engel Asael und dem Rabbi gewidmet, da der Rabbi die Gesetze Gottes nicht eingehalten hat und das Weltgefüge in Gefahr brachte. Der Erzählrahmen, der sich fantastischen Elementen bedient, besteht aus drei Kapiteln: dem Anfangs- und Schlusskapitel und dem mit Nachts unter der steinernen Brücke betitelten. Da allerdings in Prag Legende und Geschichte eine symbiotische Existenz zu haben scheint, sind auch bei Perutz die fantastischen Kapitel mit historischen Figuren durchsetzt und bilden in sich den historischen Rahmen. So sind der Rabbi Loew und der Jude Meisl historische Personen, die in Prag gelebt haben und zur Gesellschaft beigetragen haben. Sie bilden eine graduale Brücke vom Fantastischen zur Geschichtlichkeit: Es wird in drei Kapiteln Bezug auf den Juden Meisl und seinen Reichtum genommen ${ }^{2}$. Diese Kapitel befinden sich zwischen denen der Fantastik und der Geschichtlichkeit, da dokumentiert ist, dass es zu dieser Zeit einen Juden Meisl in Prag gab, der auch Kaiser Rudolf II. Hofbankier war, da dieser in ständigen Geldschwierigkeiten wegen seiner Vorliebe für die Kunst lebte. Da Meisl keine Nachfahren hatte, entschied er sich für das Mäzenatentum und nach historischen Belegen stiftete er eine Mikwa, ein Krankenhaus und ein Armenhaus. Er ließ die Judenstadt pflastern, beteiligte sich an dem Bau einer neuen Synagoge und unternahm vielerlei karitative und philanthropische Ausgaben, um das Leben der jüdischen Gemeinde zu verbessern. Seine Beteiligung am Bau des jüdischen Rathauses ist zwar umstritten, aber nicht ausgeschlossen. Er ist auf dem jüdischen Friedhof in Prag begraben. Die Ehe mit Esther, wie sie bei Perutz vorkommt, ist fiktiv, da Mordechai Meisl in erster Ehe mit Eva, Tochter des Kaufmanns in Prag und in zweiter kurzer Ehe mit Johanka, Tochter des brandenburgischen Hoffaktors und Münzmeisters verheiratet war (1572-1573). Beide Ehen blieben kinderlos und nach Meisls Tod entwickelte sich ein fast hundertjähriger Erbschaftsstreit zwischen begünstigten Verwandten (zwei Neffen) und der kaiserlichen Staatskasse, die die Gelder beschlagnahmte. Hieran schloss sich ein langwieriger Rechtsstreit zwischen den Erben und der jüdischen Gemeinde an, die ebenfalls Anspruch auf einen Teil des Erbes erhob. Dieser als historisch einzustufende Erbschaftsstreit konnte erst 1699 durch Einigung beigelegt werden. Auch wenn Esther nicht die Ehefrau des legendären Meisls war, so ist doch Perutz Namenswahl von Bedeutung: Esther geht wahrscheinlich auf die im Tanach erzählte Geschichte zurück, die historisch so nicht nachprüfbar, aber von entscheidender Bedeutung für das jüdische Volk ist, da das Erzählen dieser Geschichte fester Bestandteil des Purim-Festes ist. Esther bedeutet die Strahlende oder der Stern, was auch interpretiert werden kann als Führerin oder Retterin. Diese Rolle wird ihr in der biblischen Geschichte zugeteilt: Esther ist Waise und wird von ihrem Vetter Mordechai großgezogen. Als sie im heiratsfähigen Alter ist, wird sie mit König Ahasveros verheiratet (Esther ist hier mit dem Namen Hadassa gleichzusetzen). Mordechai, der Hofbeamter ist, bekommt später Kenntnis von dem durch Haman geplanten Völkermord der Juden im persischen Reich. Durch Esther, die nun Königin ist, gelingt es, dieses Vorhaben zu vereiteln und den vermeintlichen Todestag aller Juden in

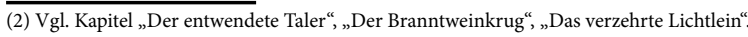


einen Tag umzuwandeln, der es den Juden erlaubt, ihre Feinde zu töten. Aus diesem einen Tag werden zwei und der Erzählung nach sterben viele Nicht-Juden. An jedem Purim-Fest wird dieses Ereignis gefeiert und Esthers Einsatz als Heldentat gelobt. Auffallend ist, dass Perutz diesen Namen aus dem Buch Esther mit dem legendären Namen Mordechai Meisl aus Prag verbindet. Geschichtlichkeit und die nicht historisch überprüfbare biblische Erzählung fallen zusammen (das heißt nicht, dass biblische Erzählungen keinen Wahrheitsgehalt besitzen, es soll nur darauf hingewiesen werden, dass es keine historischen Quellen gibt, die dies belegen können), ergänzen sich und werden innerhalb der Erzählstruktur von Perutz plausibel. Esther rettet das jüdische Volk, indem sie im Traum jede Nacht als Frau an die Seite des Kaisers gerufen wird und so dieser von Pogromen gegen die jüdische Bevölkerung absieht. Esther übernimmt auch bei Perutz die Rolle, die ihr ihr Name auferlegt, und erhält in der fiktiven Erzählstruktur einen symbolischen Gehalt.

Eine nächste Abstufung hin zur Geschichtlichkeit lässt sich in den drei Kapiteln „Der Heinrich aus der Hölle“, „Der vergessene Alchemist“ und „Die Getreuen des Kaisers“ erkennen. Diese Kapitel verweisen auf Ereignisse, die sich um den Kaiser spinnen. Sie sind fiktiv, da sie nicht beweisbar sind, aber real in dem Sinne, indem sie den schwierigen Charakter Rudolfs II. beschreiben. In die Geschichte ist eingegangen, dass Rudolf II. unter depressiven Phasen litt und auch erhebliche Schwierigkeiten im Umgang mit Frauen hatte, auch wenn er zahlreiche Geliebte hatte und uneheliche Kinder zeugte. In seinen letzten Regierungsjahren wurde immer deutlicher, dass er die Regierungsgeschäfte nicht mehr erledigen konnte. Seine depressive Anlage, die Angst vor Anschlägen, die sich mit hegenden Selbstmordplänen verbindet und der hohe Alkoholkonsum, nimmt Perutz in seinem Roman auf, um den instabilen Charakter des Kaisers zu beschreiben (Press: 1990). So lassen sich drei Phasen erkennen, die Kaisers Rudolf II. nach historischen Quellen zur Handlungsunfähigkeit leiten: Die erste Phase, ohne entscheidende Vorkommnisse, reicht bis zum Türkenkrieg von 1593. Eine zweite Phase lässt sich während dieses Krieges ersehen, die bis 1606 dauert und die den Kaiser mehr als einmal entscheidungslos ließ. Ob diese Entscheidungslosigkeiten die ersten Anzeichen eines beginnenden depressiven Krankheitsbildes oder einfach nur Kriegsratlosigkeit waren, kann nicht einfach beantwortet werden, da es an entsprechenden Daten fehlt. Die dritte Phase ist von klarer Regierungsunfähigkeit bestimmt und artikuliert sich in den letzten sechs Jahren seiner Amtszeit (Vacha: 1992). Der Charakter des Kaisers steht somit in direkter Beziehung zu den fantastischen Elementen des Romans, da so der angewandte Zauber zum Schutz der jüdischen Gemeinde legitimiert wird.

Die nächste Abstufung bilden zwei Kapitel, die scheinbar nicht einzureihen sind. Doch bei näherer Betrachtung kann man feststellen, dass das Kapitel „Das Gespräch der Hunde“ eine Verbindung zu allen im Roman auftretenden Elementen besitzt: zur Fantastik, indem auf die Kabbala rekurriert wird, damit der Held dieser Erzählepisode das Gespräch der Hunde hören kann; zu Meisl, da einer der Hunde ihm gehörte, aber nach Meisls Tod in der Judenstadt umherstreift; zum Kaiser, durch die Verhaftung des Helden, indem er neue Gesetze erlässt und schließlich in den Handlungsorten von Judenstadt und kaiserlichem Gefängnis. Auf dieser Stufe befindet sich auch das Kapitel „Der Stern des Wallenstein“, das Alchemie und Geschichtlichkeit zu verbinden versucht und die Problematik des dreißigjährigen Krieges thematisiert: katholischer und protestantischer Glauben. Dieses Kapitel ist eine weitere Annäherung an die letzten drei Kapitel, die versuchen, Geschichte „anders“ zu erzählen und sich nicht auf historische Dokumente beziehen. Diese sind „Des Kaisers Tisch“, „Die Sarabande“ und „Der Maler Barbanzio“. Diese drei Kapitel sind dadurch geprägt, dass sie vom Erzähler Stud. Med. Jakob Meisl dem auktorialen Erzähler berichtet werden. Die Kapitel beziehen sich zwar auf geschichtliche Geschehnisse, nur, dass sie im historischen Kontext nicht belegbar sind. Wahrheitsgehalt erlangen sie durch den Erzähler, der sich im Epilog zu erkennen gibt, und er, indem er zum Protagonisten und auktorialen Erzähler wird, aus der Erinnerung heraus rückblickend auf seine Jugendjahre schaut, in denen er Nachhilfeunterricht von eben jenem Studenten der Medizin Jakob Meisl bekam. Eine Verbindung zu dem Juden Meisl der Josefstadt ist nicht zu verkennen, und diese Tatsache wird im Epilog aufgegriffen: Mit dem Verschwinden des meislschen Vermögens verschwinden auch die Legenden und die alte Josefstadt verschwindet, indem sie der Sanierung zum Opfer fiel.

Die einzelnen Kapitel des Romans sind nicht chronologisch geordnet, weder in der Anordnung von fantastisch zu geschichtlich noch in ihrer chronologischen Kohärenz. Auf den ersten Blick scheint sich kein System erkennen zu lassen. Dem widerspricht allerdings die mathematische Bildung Perutz und berücksichtigt nicht, dass er mathematische Formeln und Gleichungen, zumindest aber mathematische Wiederholungen auch 
im Aufbau seiner literarischen Werke benutzte. Der vermeintliche Anschein von losen Kapiteln, die zu einem Roman verschmelzen, kann aber auch anders gelesen werden. Geht man davon aus, dass jedes Kapitel eine Formel besitzt, erhält man folgende Signaturen:

1. Kapitel: fantastische Elemente

2. Kapitel: Kommentar von Jakob Meisl

3. Kapitel: fantastisch-reale (topologisch) Elemente

4. Kapitel: Kommentar von Jakob Meisl

5. Kapitel: fiktiv-reale Elemente Bezug zum Kaiser

6. Kapitel: fiktiv-reale Elemente Bezug zu Meisl

7. Kapitel: fantastische Elemente

8. Kapitel: fantastisch-reale Elemente (Geschichtlichkeit)

9. Kapitel: Kommentar von Jakob Meisl

10. Kapitel: fiktiv-reale Elemente Bezug zum Kaiser

11. Kapitel: fiktiv-reale Elemente Bezug zu Meisl

12. Kapitel: fiktiv-reale Elemente Bezug zum Kaiser

13. Kapitel: fiktiv-reale Elemente Bezug zu Meisl

14. Kapitel: fantastische Elemente

Ersetzt man nun jede Signatur durch eine Zahl erhält man 5 Gruppen:

$1=$ fantastische Elemente

$2=$ Kommentar von Jakob Meisl

3 = fantastische-reale Elemente

4 = fiktiv-reale Elemente Bezug zum Kaiser

5 = fiktiv-reale Elemente Bezug zu Meisl

Ordnen man chronologisch diese Gruppen erhält man folgende Zahlenreihe: 1231234513245451 .

Man kann diese Zahlenreihe allerdings auch anders schreiben und so erhält man ein anderes Bild:

1 23245
1

324545
1

Versucht man nun ein System in dieser Zahlenreihe zu ersehen, kann man erkennen, dass nach der Zäsur 1 = fantastische Elemente, eine Reihe von 23245 folgt. Teilt man 23245 auf in 23 Leerstelle 2 (mal) 45 kann man die nächste Reihe nach der 1 auflösen in 32, invers gelesen und 2x45 45. Diese Sequenz hat einen Rhythmus, der sich wiederholt. Dieser Annahme entspräche auch Perutz Grundsatz, der einen Ausgleich in der Wahrscheinlichkeitsrechnung anstrebte. Dementsprechend lässt sich eine gewisse Ordnung der scheinbar losen Kapitel erkennen und Müllers Aussage müsste somit neu überdacht werden, dass «der Leser zwangsläufig den Versuch unternehmen [wird], aus der zeitlich unzusammenhängenden Folge in sich abgeschlossener Novellen eine zusammenhängende Romanhandlung zu entwerfen» (Müller 2007: 265).

\subsection{Schlussfolgerung}

Zusammenfassend kann man sagen, dass der Roman Nachts unter der steinernen Brücke verschiedene Elemente zwischen Geschichtlichkeit und Fantastik aufgreift und sie in einen narrativen fiktiv-realen Kontext setzt. Die Kapitel, in denen historische Ereignisse geschildert oder historische Personen erwähnt werden, enthalten meist einen Hinweis auf den Juden Meisl, so dass daraus abgeleitet werden kann, dass das Bindeglied die Figur Meisl entweder als fiktiv kommentierender Erzähler oder als der legendäre Mordechai Meisl des jüdischen 
Ghettos am Ende des 16. Jahrhunderts ist. Diese Annahme wird weiter unterstützt, wenn man sich die Kapitel ansieht, die dem Fantastischen gewidmet sind, das heißt, in denen das Judentum mit der Kabbala verbunden wird. Der bereits erwähnte äußere Erzählrahmen besitzt nicht nur fantastische Elemente, sondern gibt auch eine mögliche Erklärung, indem er auf die Kabbala rekurriert. Da es sich aber um einen Rahmen handelt, ist die intertextuelle Verbindung oder das Bindeglied zwischen den fantastischen Kapiteln der legendäre Mordechai Meisl, dessen Frau, die Traumfrau Kaiser Rudolfs II. ist. Diese Dreieckskonstellation ist historisch nicht belegt und verweist auf die Koexistenz zwischen Realem und Irrealen, zwischen der historischen Geschichtlichkeit und dem Fantastischen. Um aber innerhalb der Erzählstruktur das Fantastische erklären zu können, bedarf es einer Instanz im Text, die nach der Definition von Wünsch (1998) das fantastische Geschehen erklärt. Diese Rolle übernimmt der Rabbi, der die fantastischen Ereignisse nicht nur erklärt, sondern auch erschafft. Um aber in die Realität eingreifen zu können, bedarf es einer geistigen Zäsur, die durch die Verortung in den Traum erreicht wird. Traumzustände können als eine grundlegende Bedingung für fantastische Ereignisse angesehen werden, da der Traum für das Zwischenstadium zwischen Realität und Irrealität steht. Nur im Moment des Traumes können Bilder und Situationen entstehen, die im Dazwischen liegen, die zwar für den Träumenden real erlebt werden, aber dem Hier und Jetzt entrückt sind. Perutz spielt hier auf den psychischen Apparat von Freud an, und versucht anhand des Traumzustands, die drei psychischen Instanzen zu verbinden. Da diese sich auch im strukturellen Aufbau des Baums des Lebens der Kabbala wiederfinden, gelingt es Perutz die Kabbala mit der Psychologie in Verbindung zu setzen (Vgl. Hirte: 2014b).

Perutz Roman ist kein ausschließlich fantastischer, aber auch kein rein historischer Roman, sondern die verschiedenen Kapitel sind um fiktive und historische Figuren gewoben, die einen alchemistisch-fantastischen Blick auf historische Ereignisse werfen, die sich am Ende des 16. Jahrhunderts abspielten. Dank der Verknüpfung verschiedener Glaubensrichtungen, Kulturen und Epochen werden die real historischen Ereignisse unter Aspekten gelesen, die nicht erklärt werden können, da die Geschichte selbst aufgrund ihrer Verankerung in Daten und nachprüfbaren Fakten viele Fragen offenlässt und eine transkulturelle Brücke bildet, die es ermöglicht, einen historischen Kontext zu interpretieren, zu lesen und zu verstehen. In dem Sinne hatte Perutz Recht: «Es ist nichts als eine Verbeugung vor dem alten Prag» (Serke 1987: 278).

Bibliografíe:

Baecker, Dirk (2013). Beobachter unter sich. Eine Kulturtheorie. Suhrkamp.

Foucault, Michel (1978). Die Ordnung der Dinge. Suhrkamp Taschenbuch Wissenschaft.

Hirte, Ricarda (2014a). Ein Rundgang durch die Kulturgeschichte der Phantastik. Universidad Nacional de Tucumán.

Hirte, Ricarda (2014b). Die Golemfigur bei Gustav Meyrink im Kontext phantastischer Signaturen. Universidad Nacional de Tucumán.

Lachmann, Renate (2002). Erzählte Phantastik. Suhrkamp Taschenbuch Wissenschaft.

Luhmann, Niklas (1995). Kultur als historischer Begriff. In (Ders.), Gesellschaftsstruktur und Semantik. Studien zur Wissenssoziologie der modernen Gesellschaft. Bd. 4 (S. 31-54). Suhrkamp.
Müller, Hans-Harald (2007). Leo Perutz. Biographie. Paul Zsolnay Verlag.

Müller, Harro (1994). Schreibmöglichkeiten historischer Romane im 19. und 20. Jahrhundert. The Germanic Review, 69(1), 14-19. https://doi.org/10.1080/00168890.19 94.9934235

Nöth, Winfried (2002). Handbuch der Semiotik. 2. vollständig neu bearbeitete und erweiterte Ausgabe. Metzler.

Perutz, Leo (1991). Nachts unter der steinernen Brücke. Rowohlt.

Press, Volker (1990). Rudolf II. In Anton Schindling und Walter Ziegler (Hrsg.), Die Kaiser der Neuzeit. 1519-1918. Heiliges römisches Reich, Österreich, Deutschland (S. 99). C.H. Beck.

Schönhaar, Rainer (1990). Historischer Roman. In Günther Schweikle und Irmgard
Schweikle (Hrsg.), Metzler Literatur Lexikon. Begriffe und Definitionen (S. 201). Metzler.

Serke, Jürgen (1987). Böhmische Dörfer. Paul Zsolnay Verlag.

Stadtarchiv der Stadt Chur. Epidemien und Hungernöte (19.08.2021). https://web archive.org/web/20170131174614/http:// www.chur.ch/dl.php/de/0d8hg-57vu0w/ Epidemien_Hungersnoete.pdf

Todorov, Tzvetan (2013). Einführung in die fantastische Literatur. Klaus Wagenbach.

Vacha, Brigitte (1992). Die Habsburger. Eine europäische Familiengeschichte. Styria.

Wünsch, Marianne (1998). Die fantastische Literatur der frühen Moderne (1890-1930). Definition, Denkgeschichtlicher Kontext, Strukturen. Wilhelm Fink. 